\title{
Optimized temporary biventricular pacing acutely improves intraoperative cardiac output after weaning from cardiopulmonary bypass: A substudy of a randomized clinical trial
}

\author{
Daniel Y. Wang, MD, ${ }^{a}$ Marc E. Richmond, MD, ${ }^{\mathrm{b}} \mathrm{T}$. Alexander Quinn, PhD, ${ }^{\mathrm{c}}$ Ajay J. Mirani, MD, \\ Alexander Rusanov, MD, ${ }^{\mathrm{d}}$ Vinay Yalamanchi, BA, ${ }^{\mathrm{d}}$ Alan D. Weinberg, MS, \\ Santos E. Cabreriza, MBA, ${ }^{\mathrm{d}}$ and Henry M. Spotnitz, MD $^{\mathrm{d}}$
}

\begin{abstract}
Objective: Permanent biventricular pacing benefits patients with heart failure and interventricular conduction delay, but the importance of pacing with and without optimization in patients at risk of low cardiac output after cardiac surgery is unknown. We hypothesized that pacing parameters independently affect cardiac output. Accordingly, we analyzed aortic flow measured with an electromagnetic flowmeter in patients at risk of low cardiac output during an ongoing randomized clinical trial of biventricular pacing $(n=11)$ versus standard of care $(n=9)$.
\end{abstract}

\begin{abstract}
Methods: A substudy was conducted in all 20 patients in both groups with stable pacing after coronary artery bypass grafting, valve surgery, or both. Ejection fraction averaged $33 \% \pm 15 \%$, and QRS duration was $116 \pm 19 \mathrm{~ms}$. Effects were measured within 1 hour of the conclusion of cardiopulmonary bypass. Atrioventricular delay ( 7 settings) and interventricular delay (9 settings) were optimized in random sequence.
\end{abstract}

Results: Optimization of atrioventricular delay $(171 \pm 8 \mathrm{~ms})$ at an interventricular delay of $0 \mathrm{~ms}$ increased flow by $14 \%$ versus the worst setting $(111 \pm 11 \mathrm{~ms}, P<.001)$ and $7 \%$ versus nominal atrioventricular delay $(120 \mathrm{~ms}$, $P<.001)$. Interventricular delay optimization increased flow $10 \%$ versus the worst setting $(P<.001)$ and $5 \%$ versus nominal interventricular delay $(0 \mathrm{~ms}, P<.001)$. Optimized pacing increased cardiac output $13 \%$ versus atrial pacing at matched heart rate $(5.5 \pm 0.5 \mathrm{vs} 4.9 \pm 0.6 \mathrm{~L} / \mathrm{min}, P=.003)$ and $10 \%$ versus sinus rhythm $(5.0 \pm$ $0.6 \mathrm{~L} / \mathrm{min}, P=.019)$.

Conclusions: Temporary biventricular pacing increases intraoperative cardiac output in patients with left ventricular dysfunction undergoing cardiac surgery. Atrioventricular and interventricular delay optimization maximizes this benefit. (J Thorac Cardiovasc Surg 2011;141:1002-8)

Supplemental material is available online.

Biventricular pacing (BiVP) is an established therapy for congestive heart failure (CHF), and it is currently the standard of care for select patients with advanced CHF associated

From the Departments of Medicine, ${ }^{\mathrm{a}}$ Pediatrics, ${ }^{\mathrm{b}}$ and Surgery, ${ }^{\mathrm{d}}$ Columbia University, New York, NY; the Department of Physiology, Anatomy and Genetics, ${ }^{\mathrm{c}}$ University of Oxford, Oxford, United Kingdom; and the Department of Health Evidence and Policy, ${ }^{\mathrm{e}}$ Mount Sinai School of Medicine, New York, NY.

This study was funded by a grant from the National Institutes of Health (RO1 HL080152 to Dr Spotnitz). Dr Spotnitz is the George H. Humphreys, II, Professor of Surgery. Dr Wang is supported by National Institutes of Health Training Grant T32 HL007854.

Disclosures: Authors have nothing to disclose with regard to commercial support.

Read at the 2009 American Heart Association Scientific Sessions, Orlando, Fla, November 14-18, 2009. Published as an abstract: Wang DY, Richmond ME, Quinn TA, Mirani AJ, Rusanov A, Yalamanchi Y, et al. Optimized temporary biventricular pacing acutely improves intraoperative cardiac output after weaning from cardiopulmonary bypass [abstract]. Circulation. 2009;120:S800.

Received for publication April 14, 2010; revisions received June 24, 2010; accepted for publication July 6, 2010; available ahead of print Aug 30, 2010.

Address for reprints: Henry M. Spotnitz, MD, Department of Surgery, Columbia University Medical Center, 622 West 168th St, 10th floor, Vanderbilt Clinic, Rm 1010, New York, NY, 10032 (E-mail: hms2@columbia.edu).

0022-5223/\$36.00

Copyright (c) 2011 by The American Association for Thoracic Surgery doi:10.1016/j.jtcvs.2010.07.004 with left ventricular (LV) dysfunction and intraventricular conduction delay (IVCD). ${ }^{1}$ Permanent BiVP improves LV dimension and function and decreases morbidity and mortality, although it is associated with a nonresponse rate of up to $30 \% .^{2-5}$ Although the long-term benefits of BiVP are typically not appreciated until several months after implantation, hemodynamic effects of changes to pacing parameters are reflected acutely by metrics such as stroke volume, ventricular dyssynchrony, and the maximal first derivative of pressure $\left.\left(\mathrm{dP} / \mathrm{dt}_{\max }\right)\right)^{6,7}$ These properties have facilitated the study of the optimization of programmable BiVP parameters, such as atrioventricular delay (AVD) and interventricular delay (VVD), to maximize the hemodynamic benefit of BiVP and to reduce its nonresponse rate. ${ }^{8-10}$

The acute hemodynamic effects of BiVP also enable the study of temporary BiVP as a treatment for low output states after cardiac surgery. Low left ventricular ejection fraction (LVEF) is an independent risk factor for poor outcomes after cardiac surgery. ${ }^{11}$ BiVP improves hemodynamics without increasing myocardial oxygen consumption, ${ }^{7}$ and therefore it is particularly appealing as a potential therapy in patients undergoing cardiac surgery. Prior studies have assessed temporary perioperative BiVP in heterogeneous groups of patients with varying results. ${ }^{12-24}$ Moreover, the 


$$
\begin{aligned}
& \text { Abbreviations and Acronyms } \\
& \begin{aligned}
\text { AAI } & =\text { atrial pacing } \\
\text { AVD } & =\text { atrioventricular delay } \\
\text { BiPACS } & =\text { BiVP After Cardiac Surgery } \\
\text { BiVP } & =\text { biventricular pacing } \\
\text { CABG } & =\text { coronary artery bypass grafting } \\
\mathrm{CHF} & =\text { congestive heart failure } \\
\mathrm{CO} & =\text { cardiac output } \\
\mathrm{CPB} & =\text { cardiopulmonary bypass } \\
\mathrm{dP} / \mathrm{dt} & =\text { maximal first derivative of pressure } \\
\mathrm{IVCD} & =\text { intraventricular conduction delay } \\
\mathrm{LV} & =\text { left ventricle } \\
\mathrm{LVEF} & =\text { left ventricular ejection fraction } \\
\mathrm{NSR} & =\text { sinus rhythm with no pacing } \\
\mathrm{RA} & =\text { right atrium } \\
\mathrm{RV} & =\text { right ventricle } \\
\mathrm{VVD} & =\text { interventricular delay }
\end{aligned}
\end{aligned}
$$

role of optimization of temporary BiVP parameters in the perioperative setting is unclear. ${ }^{25,26}$

The BiVP After Cardiac Surgery (BiPACS) trial is a randomized clinical trial to study the effect of optimized temporary $\mathrm{BiVP}$ on cardiac output $(\mathrm{CO})$ in postoperative cardiac surgery patients with preoperative LV systolic dysfunction and an IVCD. Patients undergo BiVP optimization at multiple time points in the intraoperative and postoperative periods and are randomized to continuous optimized BiVP versus standard of care. The hypothesis underlying the $\mathrm{Bi}$ PACS trial is that CO will increase $15 \%$ in patients undergoing temporary BiVP. In this substudy of the BiPACS trial, we hypothesized that optimization of pacing parameters would increase CO. Accordingly, we assessed the contribution of AVD and VVD optimization to the effect of BiVP optimization in the intraoperative period after separation from cardiopulmonary bypass (CPB) and evaluated the effect of optimized BiVP on CO compared with atrial pacing (AAI) and with sinus rhythm with no pacing (NSR).

\section{MATERIALS AND METHODS \\ BiPACS Trial Study Population}

The study protocol was approved by the Columbia University Medical Center Institutional Review Board. Adult patients undergoing elective cardiac surgery on CPB were screened for eligibility to enroll in the BiPACS trial. All patients provided written informed consent. Recruitment was done before the day of the operation by qualified and trained study coordinators and investigators on the study team with permission of the attending surgeon. Inclusion criteria included the following: preoperative CHF, LVEF of $40 \%$ or less, and a QRS duration of $100 \mathrm{~ms}$ or greater or patients undergoing combined mitral and aortic valve surgery. LVEF and QRS criteria were liberalized from values of $35 \%$ and $120 \mathrm{~ms}$, respectively, in the original protocol. Exclusion criteria included the following: atrial fibrillation, second- or third-degree atrioventricular block, congenital heart disease, intracardiac shunts, or heart rate of greater than 120 beats/min after separation from CPB. Preoperative data obtained by means of chart review included the following: LVEF, as measured by means of echocardiographic or left ventriculogram analysis; heart rhythm, QRS duration, and intraventricular blocks from electrocardiographic tracings; the type of operation performed; and demographic characteristics. The BiPACS trial is ongoing, and end points will not be examined until 212 patients have been randomized.

\section{Study Design and Optimization Protocol}

Patients in the BiPACS trial are randomized to the 2 treatment groups at the end of phase 1 (within 1 hour of the conclusion of cardiopulmonary bypass) by using randomly permuted blocks of 4,6 , and 8 to avoid imbalances that can occur with simple randomization. A treatment allocation ratio of 1:1 was used; each group will be of equal size. The phase 1 testing described here occurs in all patients before randomization. Optimization of AVD, ventricular pacing site, and VVD are tested in random sequences. Randomization and testing sequences are determined based on forms in sealed envelopes that are not opened until needed. These forms were prepared before enrollment of the first patient. Before separation from CPB, temporary epicardial pacing leads were sewn to the right atrial (RA) appendage, anterior right ventricle (RV), and 2 randomized sites of 6 possible sites on the LV. One of the LV leads (LV1) was placed at the basal LV at either the obtuse margin, circumflex, or posterior regions; the second LV lead (LV2) was placed at either the midinferomedial, midinferolateral, or apical LV. Data from BiVP using LV1 were analyzed in this study. The leads were attached to a Medtronic InSync III permanent biventricular pacemaker (Medtronic, Inc, Minneapolis, Minn) mounted in an external housing unit, and their sensing and pacing functions were tested and confirmed. An appropriately sized electromagnetic flow probe (Carolina Medical Electronics, East Bend, NC) was placed on the ascending aorta. After separation from CPB and establishment of stable inotrope and vasopressor dosing, the BiVP optimization protocol was initiated. The pacing rate was set at 90 beats/min or at 10 beats/min greater than the patient's intrinsic heart rate if greater than 90 beats/min to ensure atrial capture up to a maximum of 120 beats/min. These heart rates were selected empirically. A wider range of heart rates is studied in phase 3 of the BiPACS trial, including cardiac resynchronization therapy at the intrinsic heart rate.

Real-time aortic volume flow, echocardiograms, and arterial pressure signals were collected with an analog-to-digital converter (PowerLab; ADInstruments, Inc, Milford, Mass) and recorded on a personal computer (iMac; Apple Computer, Inc, Cupertino, Calif; Figure 1). CO was measured by integrating aortic volume flow tracings over 1 respiratory cycle with MacLab software (ADInstruments, Inc) and custom-designed routines in Matlab (The MathWorks, Inc, Natick, Mass).

BiVP optimization was performed by optimizing AVD, followed by VVD. All pacing settings during optimization were conducted over 10-second intervals and were tested twice. The use of a rapid optimization protocol measuring changes in cardiac mechanics over brief intervals has been described previously. ${ }^{14,27}$ AVD optimization was performed during sequential RA BiVP, with a VVD of $0 \mathrm{~ms}$. AVD was varied in 30-ms increments, ranging from 90 to $270 \mathrm{~ms}$, in randomized order. AVDs that were longer than the patient's intrinsic paced AVD were not tested. The AVD yielding the highest CO was selected as the optimum AVD. AVD optimization data from a representative patient are shown in Figure 2. VVD optimization was then performed with the optimum AVD by varying the VVD by $20-\mathrm{ms}$ increments, ranging from $-80 \mathrm{~ms}$ (LV first) to $+80 \mathrm{~ms}$ (RV first) in randomized order. CO as a function of VVD was plotted and the VVD yielding the highest $\mathrm{CO}$ was selected as the optimum VVD (Figure 3), thereby defining the optimum BiVP parameters for the patient. Optimized BiVP was then compared with RA pacing (AAI mode) at the same heart rate and with NSR with no pacing, in randomized order, and over 30-second intervals. The aortic flow probe was then removed, and the temporary pacing leads were externalized for further BiVP optimization in subsequent phases of the BiPACS trial. 


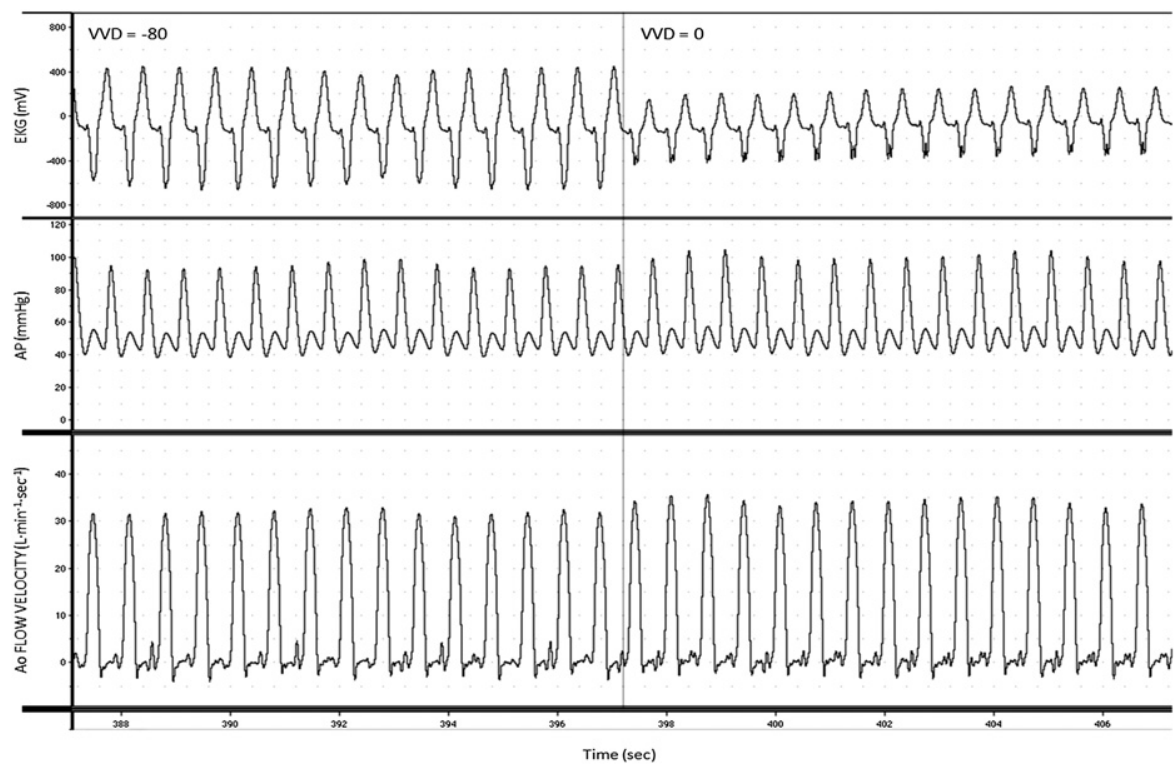

FIGURE 1. Intraoperative recordings from a representative patient displaying changes in electrocardiograms $(E K G)$, arterial pressure ( $A P)$, and aortic $(A o)$ flow velocity during biventricular pacing optimization. $V V D$, Interventricular delay.

\section{Statistical Analysis}

For AVD and VVD optimization data and for the comparison among optimized BiVP, AAI, and NSR, descriptive statistics were calculated for each group. Differences among multiple groups (3) were tested by using blocked 1 -way analysis of variance. Post hoc comparisons to assess pairwise differences between groups were performed with the Tukey test adjusted for multiple comparisons. Differences between 2 groups were tested by using a 2-way paired Student's $t$ test. Statistical analysis was performed with SAS 9.1 software (SAS Institute, Inc, Cary, NC).

\section{RESULTS}

Patient flow for the BiPACS trial and this substudy covers recruitment from April 1, 2007, to June 2, 2009. The number of patients screened was 2261 , and 60 were enrolled. Thirtythree patients received the intended testing in phase 1 and were subsequently randomized to BiVP (experimental

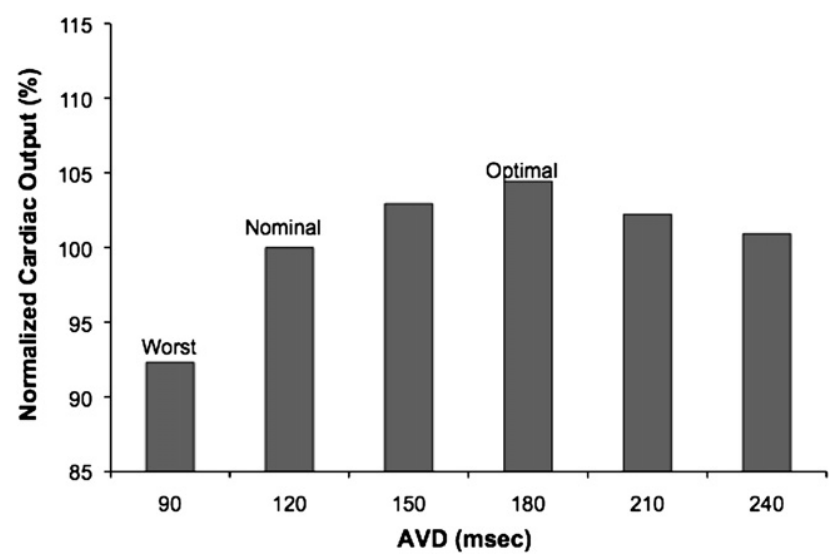

FIGURE 2. Cardiac output versus atrioventricular delay $(A V D)$ from a representative patient during AVD optimization. group) or standard of care (control group). There were no adverse events during phase 1 . The number of these patients for whom the primary end point was measured was 13 in the BiVP group and 13 in the standard-of-care group. The study has not been completed, however, and therefore no analysis of primary and secondary outcomes has been done, and the present study only describes the results for phase 1 . Accordingly, the data after randomization and the period of follow-up are not relevant and are not summarized.

Among the 33 patients who received phase 1 testing in the BiPACS trial, 13 were eliminated from this substudy because of frequent ventricular ectopy, an intra-aortic balloon pump, or noisy aortic flow tracings. Ultimately, of the 20 patients included in this substudy, there were 13 without second- or third-degree atrioventricular block

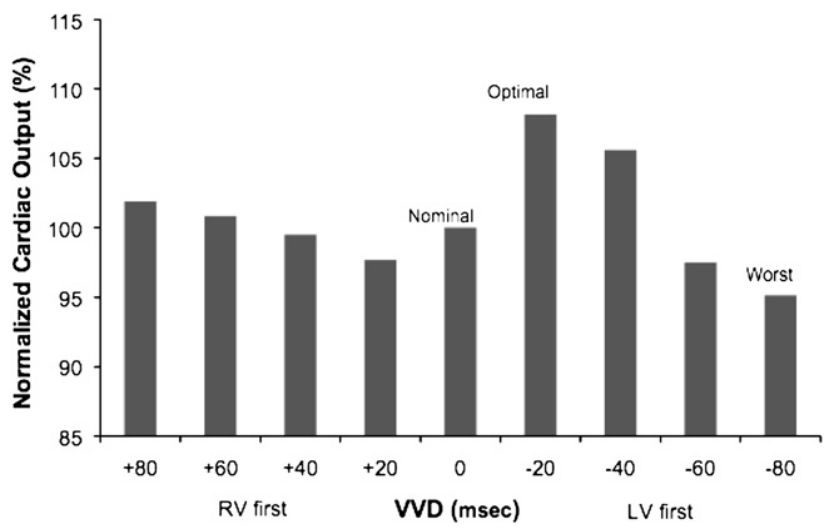

FIGURE 3. Cardiac output versus interventricular delay $(V V D)$ from a representative patient during VVD optimization. $L V$, Left ventricle; $R V$, right ventricle. 
who completed comparison of optimized BiVP, AAI, and NSR. Baseline clinical characteristics are shown in Table E1.

The majority of patients underwent valvular surgery, including double-valve cases, as well as combination valve and coronary artery bypass grafting (CABG) surgery. Average age was $67 \pm 12$ (standard deviation) years, and 75\% were male. LVEF averaged $33 \% \pm 15 \%$, and average QRS duration was $116 \pm 19 \mathrm{~ms}$. Nine patients underwent combined CABG and aortic valve replacement, mitral valve replacement, or both. Five underwent combined aortic and mitral valve surgery, 3 underwent aortic surgery alone, and 3 underwent isolated CABG.

Results of AVD and VVD optimization are shown in Figure 4. The intrinsic paced AVD was greater than 150 $\mathrm{ms}$ in all patients and greater than $270 \mathrm{~ms}$ in 10 patients. The mean optimum AVD was $171 \pm 8$ versus $111 \pm 11$ $\mathrm{ms}$ (standard error of the mean) for the mean worst AVD $(P<.001)$. The optimum AVD was greater than $150 \mathrm{~ms}$ in 10 patients and greater than $120 \mathrm{~ms}$ in all but 2 patients. Comparison of mean $\mathrm{CO}$ for the optimized, worst, and nominal (120-ms) AVD settings showed significant differences among groups $(P<.001)$. In pairwise comparisons mean $\mathrm{CO}$ was different in both the optimum and worst groups compared with that at an AVD of $120 \mathrm{~ms}$. BiVP with the optimum AVD differed from the worst AVD $(P<.001)$, with a mean increase in CO of $14 \%$ (range, $2 \%-34 \%$ ). The optimal AVD differed from an AVD of $120 \mathrm{~ms}(P<.001)$, with a mean increase in $\mathrm{CO}$ of $7 \%$ (range, $0 \%-34 \%$ ).

VVD optimization after AVD optimization yielded significant differences in $\mathrm{CO}$ when comparing the optimum, worst, and nominal $(0-\mathrm{ms})$ VVD settings $(P<.001$, Figure 4$)$. In pairwise comparisons both the optimum and worst VVDs differed from the nominal VVD. BiVP with the optimum VVD differed from the worst VVD $(P<.001)$, increasing

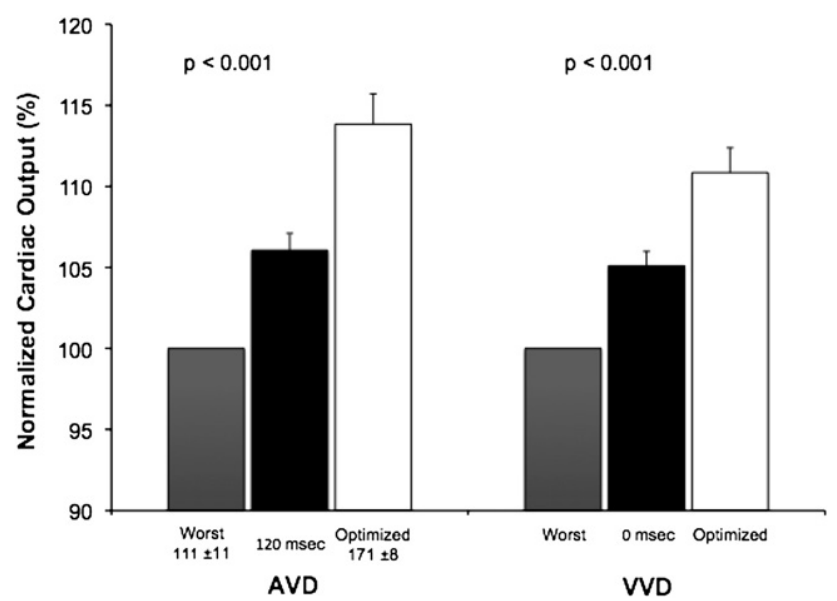

FIGURE 4. Biventricular pacing optimization profiles for atrioventricular delay $(A V D)$ and interventricular delay $(V V D)$. Mean cardiac outputs are normalized to a scale of 100 , with the worst parameter as the reference group. Error bars depict 1 standard error of the mean.
CO by $10 \%$ (range, $4 \%-29 \%$ ), and from the nominal $\operatorname{VVD}(P<.001)$, increasing CO by $5.0 \%$ (range, $0 \%-29 \%$ ).

Distributions of optimal and worst AVDs and VVDs are shown in Figures E1 and E2, respectively. An AVD of 90 ms yielded the lowest $\mathrm{CO}$ in the majority of patients. In all but 2 patients, the optimal AVD was greater than $120 \mathrm{~ms}$, and in 3 patients an AVD of $120 \mathrm{~ms}$ was the worst setting. VVD optimization resulted in a pattern of optimum and worst VVD settings, ranging from RV-first to LV-first pacing, likely reflecting heterogeneity in the types of IVCD among patients. In 2 patients the nominal VVD yielded the lowest CO.

Comparison of optimized BiVP with AAI and NSR is shown in Figure 5. The differences among the 3 groups were significant $(P=.003)$, as were pairwise comparisons between optimized BiVP versus AAI and NSR $(P=.003$ and .019 , respectively). Optimized BiVP resulted in an increase in mean $\mathrm{CO}$ by $13 \%$ versus $\mathrm{AAI}$ at the same heart rate $(\mathrm{CO}$ of $5.5 \pm 0.6$ vs $4.9 \pm 0.6 \mathrm{~L} / \mathrm{min}$ ) and by $10 \%$ versus NSR $(5.0 \pm 0.5 \mathrm{~L} / \mathrm{min})$. The paced heart rate was greater in the BiVP and AAI groups ( $97 \pm 3$ beats/min) compared with that seen in the NSR group $(80 \pm 4$ beats/min, $P<.001)$.

\section{DISCUSSION}

Defining the best modality and method for permanent BiVP optimization to improve BiVP response rates and heart failure outcomes is an active area of investigation. To our knowledge, the BiPACS trial is the first randomized clinical trial to assess the role of temporary BiVP optimization at multiple time points in the perioperative cardiac surgery setting. In this substudy, which focused on intraoperative BiVP in the immediately post-CPB period, we found that BiVP optimization increased $\mathrm{CO}$ compared with both AAI and NSR (no pacing), with significant contributions from optimization of both AVD and VVD (Figure 4). The difference in $\mathrm{CO}$ between the best and worst

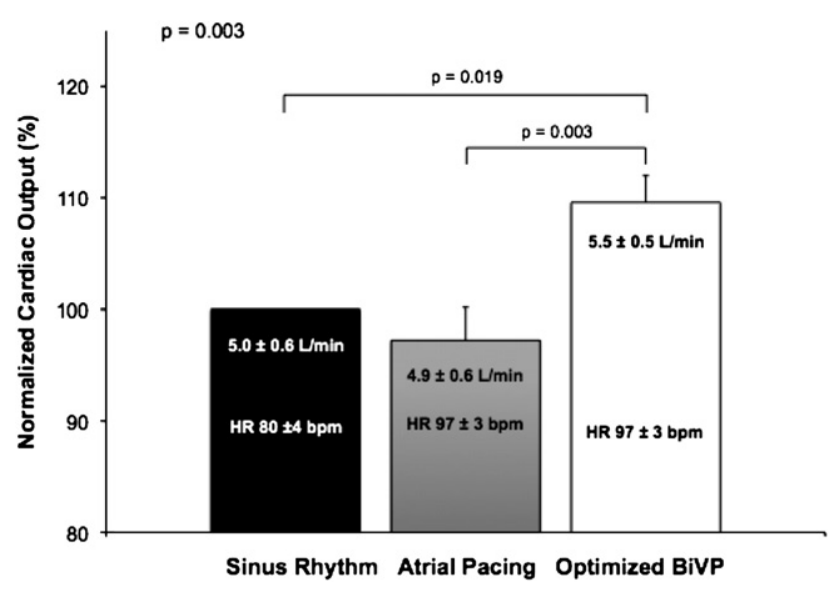

FIGURE 5. Comparison of cardiac output with optimized biventricular pacing $(B i V P)$ versus right atrial pacing or sinus rhythm. Error bars depict 1 standard error of the mean. $H R$, Heart rate. 
settings was considerable, ranging as high as $34 \%$ in an individual patient. Moreover, in 5 patients programming a nominal AVD or VVD resulted in the least effective BiVP setting, which supports a rationale for routine optimization in all patients undergoing temporary BiVP.

AVD optimization represents a balance between optimizing LV filling and atrial emptying and minimizing diastolic mitral regurgitation. Nominal out-of-the-box AVD settings are typically programmed to short intervals, such as 120 $\mathrm{ms}$, to ensure biventricular capture. However, empiric use of short AVDs might routinely underestimate the optimal AVD. ${ }^{28}$ Longer intrinsic paced AVDs in the post-CPB period allowed for testing of AVDs greater than or equal to $240 \mathrm{~ms}$ in the majority of patients. AVD optimization alone resulted in a $14 \%$ increase in CO between the best and worst settings and a 7\% increase compared with an AVD of 120 ms. In all but 2 patients, the optimum AVD was longer than $120 \mathrm{~ms}$ (see Figure E1). Indeed, in the immediate post-CPB period, factors contributing to impaired diastolic function, such as myocardial ischemia and edema, might necessitate the use of longer AVDs in patients with impaired LV function. In this study atrio-BiVP was achieved by pacing from the RA. RA pacing alters interatrial delay and the timing of left atrial-LV contraction, which implies that the optimum AVD would differ in right atrially paced, biatrially paced, and atrially sensed BiVP modes. ${ }^{29,30}$

Modulation of VVD has been shown to reduce ventricular dyssynchrony and improve hemodynamic parameters. ${ }^{9,10}$ In the present study VVD optimization increased CO by $10 \%$ and 5\% compared with the worst and nominal settings, respectively, underscoring the additional benefit of VVD optimization, even after AVD has been optimized. Whether the sequence in which AVD and VVD are optimized affects the determination of optimized BiVP parameters is uncertain and warrants further study.

Optimized BiVP improved CO by 13\% compared with $\mathrm{AAI}$ at the same heart rate, indicating that the mechanism of hemodynamic benefit in BiVP was not explained solely by an increased paced rate compared with the patient's intrinsic sinus rate (Figure 5). This finding is consistent with previous studies of temporary BiVP. ${ }^{13,17}$

In this study the mean preoperative LVEF $(33.4 \%)$ was higher and the mean QRS (115.7 ms) was narrower than in permanent BiVP trials (see Table E1) ${ }^{2-5}$ Although the criteria for permanent BiVP implantation are established, ${ }^{1}$ the predictors of acute response to temporary perioperative BiVP have yet to be defined and are an area of ongoing investigation. Cardiac surgery and extracorporeal circulation cause transient myocardial depression and edema and might exacerbate conduction abnormalities. Patients with preexisting LV dysfunction are among the highest-risk cardiac surgery patients and are an appropriate group in which to assess the benefit of temporary BiVP. Recent evidence suggests that ambulatory patients with $\mathrm{CHF}$ and narrow QRS complexes do not benefit from permanent BiVP, despite exhibiting echocardiographic evidence of dyssynchrony. ${ }^{31}$ Whether this applies to temporary perioperative BiVP remains to be seen, especially in a heterogeneous population of ischemic and valvular cardiac surgery patients.

The mechanism by which BiVP acutely reduces dyssynchrony and improves hemodynamics is not fully understood, particularly in perioperative ventricular failure. We have previously described animal models of acute rightand left-sided heart failure and found BiVP to be beneficial in those settings. ${ }^{32-34}$ The mechanism of action appears to be synchronization of pressure development across the interventricular septum, which allows the less impaired ventricle to assist the failing one. ${ }^{34}$ These findings provide a further rationale for studying temporary BiVP in the perioperative setting and will serve as a guide for future studies examining the mechanism behind the effect demonstrated in this study.

Another area of uncertainty lies in selecting the best metric by which to assess BiVP optimization, including measures of ventricular dyssynchrony, mitral inflow, stroke volume, and $\mathrm{dP} / \mathrm{dt}_{\max }$. The present study optimized BiVP based on $\mathrm{CO}$, which is an important short-term end point for end-organ perfusion, particularly in critically ill postoperative patients with low output states. Further study is needed to evaluate the relationship between hemodynamic responses to temporary $\mathrm{BiVP}$, changes in ventricular synchrony, and patients' outcomes.

Outcomes data, including morbidity, mortality, intensive care unit length of stay, and hospital costs are important secondary objectives of the BiPACS trial. Although the absolute changes in $\mathrm{CO}$ reported here during BiVP are relatively modest, the clinical effect might be important if amplified by a reduced requirement for $\beta$-agonists and vasopressors, with secondary improvements in peripheral organ function, fluid requirements, and incidence of arrhythmias.

Accumulating data indicate differences between patients in the BiPACS trial and those undergoing permanent BiVP for chronic heart failure. The optimal BiPACS trial protocol changes over time, and the effect also changes, with benefits being primarily rate related on postoperative day 1 but primarily related to stroke volume increases in the early post$\mathrm{CPB}$ period. Absolute increases in $\mathrm{CO}$ during $\mathrm{BiVP}$ also tend to be larger in BiPACS trial patients with higher preoperative ejection fractions, contradicting observations made in patients with chronic heart failure. ${ }^{35}$ These differences suggest that the effects of BiVP in BiPACS trial are primarily mediated through effects on reversible myocardial injury rather than chronic dysfunction. These differences provide a rationale for expanding the selection of patients in our trial beyond the current recommendations for permanent BiVP. Digital transesophageal echocardiographic data capable of defining changes in regional wall motion abnormalities is an important research goal of the BiPACS trial. 
The BiPACS trial is being done under an Investigational Device Exemption from the Food and Drug Administration because, as this is written, there is no biventricular pacemaker approved for temporary pacing in the operating room. Similarly, until our trial is completed, we will not have definitive information regarding optimum LV lead locations. Empirically, temporary BiVP can be implemented by adding a temporary bipolar lead configuration to the lateral basal segment of the LV. These leads can be connected to the output terminals of a standard external temporary pacemaker in conjunction with bipolar temporary RV leads connected to the same terminals. This will result in BIVP with a VVD of zero. AVD can then be optimized empirically by using mean aortic pressure or aortic flow criteria. Our current observations indicate that the optimum AVD might be as long as $300 \mathrm{~ms}$ in the early postoperative period, particularly in patients with atrial latency in excess of $100 \mathrm{~ms}$ (Rusanov et al, unpublished data).

In conclusion, optimization of temporary BiVP improves $\mathrm{CO}$ in patients undergoing cardiac surgery with preoperative evidence of LV systolic dysfunction and an IVCD. Individualized optimization of AVD and VVD each contributes to the overall benefit of optimized BiVP, and optimization should be considered a routine step in temporary BiVP protocols. Temporary BiVP to treat low output states after cardiac surgery is a promising area of investigation. Further studies with larger numbers of patients will better define patient selection criteria and refine optimization techniques.

We thank the BiPACS trial patients and investigators and the Columbia University Division of Cardiothoracic Surgery for their participation. Lauren Bedrosian, our study coordinator 20092010, and Giselle Brown provided important assistance with manuscript preparation.

\section{References}

1. Epstein AE, DiMarco JP, Ellenbogen KA, Estes NA 3rd, Freedman RA, Gettes LS, et al. ACC/AHA/HRS 2008 guidelines for device-based therapy of cardiac rhythm abnormalities. Circulation. 2008;117:e350-408.

2. Cazeau S, Leclercq C, Lavergne T, Walker S, Varma C, Linde C, et al. Effects of multisite biventricular pacing in patients with heart failure and intraventricular conduction delay. $N$ Engl J Med. 2001;344:873-80.

3. Abraham WT, Fisher WG, Smith AL, Delurgio DB, Leon AR, Loh E, et al. Cardiac resynchronization in chronic heart failure. $N$ Engl J Med. 2002;346: 1845-53.

4. Bristow MR, Saxon LA, Boehmer J, Krueger S, Kass DA, De Marco T, et al. Cardiac-resynchronization therapy with or without an implantable defibrillator in advanced chronic heart failure. N Engl J Med. 2004;350:2140-50.

5. Cleland JG, Daubert JC, Erdmann E, Freemantle N, Gras D, Kappenberger L, et al. The effect of cardiac resynchronization on morbidity and mortality in heart failure. $N$ Engl J Med. 2005;352:1539-49.

6. Kass DA, Chen CH, Curry C, Talbot M, Berger R, Fetics B, et al. Improved left ventricular mechanics from acute VDD pacing in patients with dilated cardiomyopathy and ventricular conduction delay. Circulation. 1999;99:1567-73.

7. Nelson GS, Berger RD, Fetics BJ, Talbot M, Spinelli JC, Hare JM, et al. Left ventricular or biventricular pacing improves cardiac function at diminished energy cost in patients with dilated cardiomyopathy and left bundle-branch block. Circulation. 2000;102:3053-9.

8. Stellbrink C, Breithardt OA, Franke A, Sack S, Bakker P, Auricchio A, et al. Impact of cardiac resynchronization therapy using hemodynamically optimized pacing on left ventricular remodeling in patients with congestive heart failure and ventricular conduction disturbances. J Am Coll Cardiol. 2001;38:1957-65.

9. León AR, Abraham WT, Brozena S, Daubert JP, Fisher WG, Gurley JC, et al. Cardiac resynchronization with sequential biventricular pacing for the treatment of moderate-to-severe heart failure. J Am Coll Cardiol. 2005;46:2298-304.

10. van Gelder BM, Bracke FA, Meijer A, Lakerveld LJ, Pijls NH. Effect of optimizing the $\mathrm{VV}$ interval on left ventricular contractility in cardiac resynchronization therapy. Am J Cardiol. 2004;93:1500-3.

11. Yau TM, Fedak PW, Weisel RD, Teng C, Ivanov J. Predictors of operative risk for coronary bypass operations in patients with left ventricular dysfunction. J Thorac Cardiovasc Surg. 1999;118:1006-13.

12. Saxon LA, Kerwin WF, Cahalan MK, Kalman JM, Olgin JE, Foster E, et al. Acute effects of intraoperative multisite ventricular pacing on left ventricular function and activation/contraction sequence in patients with depressed ventricular function. J Cardiovasc Electrophysiol. 1998;9:13-21.

13. Weisse U, Isgro F, Werling $\mathrm{CH}$, Lehmann A, Saggau W. Impact of atriobiventricular pacing to poor left-ventricular function after CABG. Thorac Cardiovasc Surg. 2002;50:131-5.

14. Berberian G, Quinn TA, Kanter JP, Curtis LJ, Cabreriza SE, Weinberg AD, et al Optimized biventricular pacing in atrioventricular block after cardiac surgery. Ann Thorac Surg. 2005;80:870-5.

15. Dzemali O, Bakhtiary F, Dogan S, Wittlinger T, Moritz A, Kleine P. Perioperative biventricular pacing leads to improvement of hemodynamics in patients with reduced left-ventricular function-interim results. Pacing Clin Electrophysiol. 2006;29:1341-5.

16. Schmidt C, Frielingsdorf J, Debrunner M, Tavakoli R, Genoni M, Straumann E, et al. Acute biventricular pacing after cardiac surgery has no influence on regional and global left ventricular systolic function. Europace. 2007;9:432-6.

17. Pichlmaier M, Bagaev E, Lichtenberg A, Teebken O, Klein G, Niehaus M, et al. Four-chamber pacing in patients with poor ejection fraction but normal QRS durations undergoing open-heart surgery. Pacing Clin Electrophysiol. 2008;31: 184-91.

18. Muehlschlegel JD, Peng YG, Lobato EB, Hess PJ Jr, Martin TD, Klodell CT Jr. Temporary biventricular pacing post-cardiopulmonary bypass in patients with reduced ejection fraction. J Card Surg. 2008;23:324-30.

19. Eberhardt F, Heringlake M, Massalme MS, Dyllus A, Misfeld M, Sievers HH, et al. The effect of biventricular pacing after coronary artery bypass grafting: a prospective randomized trial of different pacing modes in patients with reduced left ventricular function. J Thorac Cardiovasc Surg. 2009;137:1461-7.

20. Foster AH, Gold MR, McLaughlin JS. Acute hemodynamic effects of atriobiventricular pacing in humans. Ann Thorac Surg. 1995;59:294-300.

21. Flynn MJ, McComb JM, Dark JH. Temporary left ventricular pacing improves haemodynamic performance in patients requiring epicardial pacing post cardiac surgery. Eur J Cardiothorac Surg. 2005;28:250-3.

22. Hajiseyedjavadi O, Pasque M, Moon M, Damiano R, Mucha T, Hebert K, et al Coronary artery bypass grafting and biventricular pacing efficacy: do past trials dictate a change in future practice? J Thorac Cardiovasc Surg. 2006;132:974-5.

23. Evonich RF, Stephens JC, Merhi W, Dukkipati S, Tepe N, Shannon F, et al. The role of temporary biventricular pacing in the cardiac surgical patient with severely reduced left ventricular systolic function. J Thorac Cardiovasc Surg. 2008;136:915-21.

24. Flynn M, Dark JH, McComb JM. Biventricular pacing after cardiac surgery. $J$ Thorac Cardiovasc Surg. 2009;138:259-60.

25. Dekker AL, Phelps B, Dijkman B, van der Nagel T, van der Veen FH, Geskes GG et al. Epicardial left ventricular lead placement for cardiac resynchronization therapy: optimal pace site selection with pressure-volume loops. J Thorac Cardiovasc Surg. 2004;127:1641-7.

26. Hanke T, Misfeld M, Heringlake M, Schreuder JJ, Wiegand UK, Eberhardt F. The effect of biventricular pacing on cardiac function after weaning from cardiopulmonary bypass in patients with reduced left ventricular function: a pressurevolume loop analysis. J Thorac Cardiovasc Surg. 2009;138:148-56.

27. Rabkin DG, Cabreriza SE, Curtis LJ, Mazer SP, Kanter JP, Weinberg AD, et al. Load dependence of cardiac output in biventricular pacing: right ventricular pressure overload in pigs. J Thorac Cardiovasc Surg. 2004;127:1713-22.

28. Gold MR, Niazi I, Giudici M, Leman RB, Sturdivant JL, Kim MH, et al A prospective comparison of AV delay programming methods for hemodynamic optimization during cardiac resynchronization therapy. J Cardiovasc Electrophysiol. 2007;18:490-6.

29. Cha YM, Nishimura RA, Hayes DL. Difference in mechanical atrioventricular delay between atrial sensing and atrial pacing modes in patients with hypertrophic and dilated cardiomyopathy: an electrical hemodynamic catheterization study. J Interv Card Electrophysiol. 2002;6:133-40. 
30. Bernheim A, Ammann P, Sticherling C, Burger P, Schaer B, Brunner-La Rocca HP, et al. Right atrial pacing impairs cardiac function during resynchronization therapy: acute effects of DDD pacing compared to VDD pacing. J Am Coll Cardiol. 2005;45:1482-7.

31. Beshai JF, Grimm RA, Nagueh SF, Baker JH 2nd, Beau SL, Greenberg SM, et al. Cardiac-resynchronization therapy in heart failure with narrow QRS complexes. N Engl J Med. 2007;357:2461-71.

32. Quinn TA, Berberian G, Cabreriza SE, Maskin LJ, Weinberg AD, Holmes JW, et al. Effects of sequential biventricular pacing during acute right ventricular pressure overload. Am J Physiol Heart Circ Physiol. 2006;291:H2380-7.
33. Berberian G, Quinn TA, Cabreriza SE, Garofalo CA, Barrios DM, Weinberg AD, et al. Load dependence of cardiac output in biventricular pacing: left ventricular volume overload in pigs. J Thorac Cardiovasc Surg. 2006;131:666-70.

34. Quinn TA, Cabreriza SE, Richmond ME, Weinberg AD, Holmes JW, Spotnitz HM. Simultaneous variation of ventricular pacing site and timing with biventricular pacing in acute ventricular failure improves function by interventricular assist. Am J Physiol Heart Circ Physiol. 2009;297:H2220-6.

35. Spotnitz MD, Wang DY, Cabreriza S, Rusanov A, Cheng B, Quinn A, et al. Does ejection fraction predict response to biventricular pacing after cardiac surgery [abstract]? ASAIO J. 2010;56:102. 


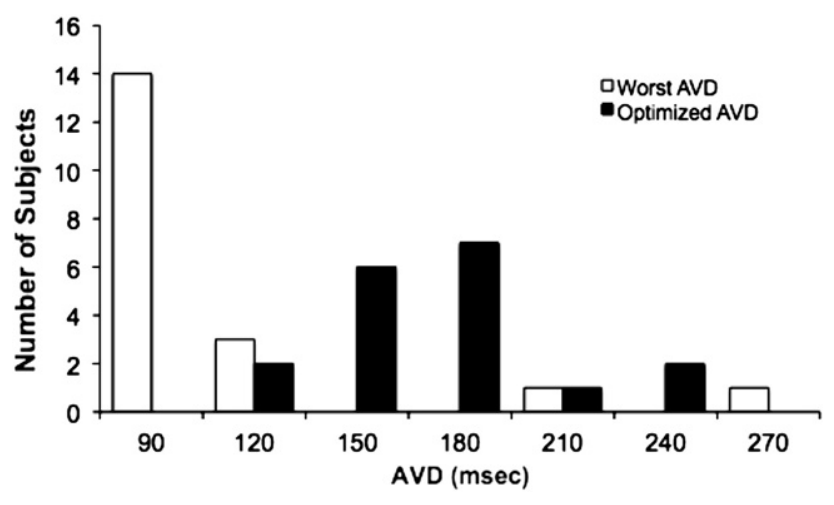

FIGURE E1. Distribution of optimal and worst atrioventricular delay $(A V D)$ settings determined during AVD optimization.

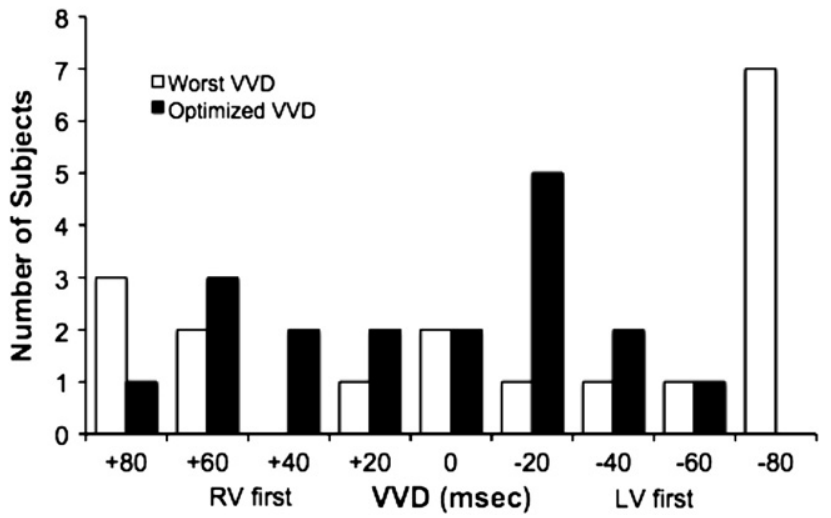

FIGURE E2. Distribution of optimal and worst interventricular delay $(V V D)$ settings determined during VVD optimization. $L V$, Left ventricle; $R V$, right ventricle.

TABLE E1. Baseline characteristics

\begin{tabular}{lc}
\hline No. of patients & \\
Total & 20 \\
Optimization analysis & 20 \\
Optimized BiVP vs AAI vs sinus rhythm & 13 \\
Age $(\mathrm{y} \pm \mathrm{SD})$ & $67.6 \pm 12.2$ \\
Ejection fraction $(\% \pm \mathrm{SD})$ & $33.4 \pm 15.4$ \\
QRS duration (ms $\pm \mathrm{SD})$ & $115.7 \pm 19.1$ \\
Male sex $(\%)$ & 75 \\
Type of operation & \\
CABG/AVR, CABG/MVR, CABG/AVR/MVR & 5 \\
AVR/MVR & 3 \\
CABG & 3 \\
AVR & 5 \\
\hline BiVP, Biventricular pacing; $A A I$, atrial pacing; $S D$, standard deviation; $C A B G$, coro- \\
nary artery bypass grafting; $A V R$, aortic valve replacement; $M V R$, mitral valve replace- \\
ment or repair.
\end{tabular}

